\title{
Multiple cargo deliveries of growth factors and antimicrobial peptide using biodegradable nanopolymer as a potential wound healing system
}

This article was published in the following Dove Medical Press journal: International Journal of Nanomedicine

\section{Amritha Vijayan \\ Pinky Prabha James \\ CK Nanditha \\ GS Vinod Kumar}

Chemical Biology, Nano Drug Delivery Systems (NDDS), Bio-Innovation Center (BIC), Rajiv Gandhi Centre for Biotechnology, Thiruvananthapuram, Kerala 695014, India

Correspondence: GS Vinod Kumar Chemical Biology, Nano Drug Delivery Systems (NDDS), Bio-Innovation Center (BIC), Rajiv Gandhi Centre for Biotechnology, Poojappura, Thycaud PO, Thiruvananthapuram, Kerala 6950I4, India

$\mathrm{Tel}+9 \mid$ 47| 2529526

Fax +9| 47। 2348096

Email gsvinod@rgcb.res.in
Background: Treatment of wounds with the help of nanoparticles (NPs) is more effective and superior in comparison to traditional wound healing methods as it protects and sustains active drug release at the wound site thus enhancing the safety of the drug and reducing the possibility of side effects. The advantages of this method are the possibility of allowing a reduction in administered dose, limiting toxicity levels to the minimum, and increasing safety of topical delivery of the drug.

Materials and methods: We report the synthesis of a novel poly (lactic-co-glycolic acid) (PLGA) NP-based multicargo delivery system for growth factors and antimicrobial peptide. Growth factors vascular endothelial growth factor (VEGF) and basic fibroblast growth factor (bFGF) were entrapped in PLGA NPs by solvent diffusion method and an antimicrobial peptide (K4) was conjugated to the NP by carbodiimide chemistry. The developed multiple cargo delivery systems with growth factors (VEGF and bFGF) and an antimicrobial peptide (K4) were investigated and optimized for potential wound healing.

Results: The system showed a sustained release of growth factors and was evaluated for cytotoxicity by MTT and live/dead assay, which revealed that the bioactivity of the growth factor-entrapped NPs was higher than that of free growth factors, and it also induced enhanced cell proliferation in vitro.

Conclusion: The development of a system for the codelivery of growth factors (VEGF and bFGF) and an antimicrobial peptide (K4) was investigated for potential wound healing application. The entrapment of growth factors with very high efficiency is an advantage in this method along with its sustained release from the nanoparticulate system, which will enhance the angiogenesis. Our system also displayed broad-spectrum antimicrobial activity against both gram-positive and gram-negative bacteria.

Keywords: growth factors, VEGF, bFGF, PLGA, antimicrobial peptides

\section{Introduction}

Treatment of wounds with the help of nanoparticles (NPs) is more effective and superior in comparison to traditional wound healing methods as it protects and sustains active drug release at the wound site, thus enhancing the safety of the drug and reducing the possibility of side effects. This is enabled by the fact that the entire amount of the encapsulated drug does not come into direct contact with the skin surrounding the wound site in one instance. The wound is instead exposed to it gradually in small doses. This process also helps to prevent biologic degradation of loaded drugs, thus ensuring enhanced stability and sustained release. The advantages of this method are the possibility of allowing a reduction in administered dose, limiting toxicity levels 
to the minimum, and increasing safety of topical delivery of the drug. ${ }^{1,2}$

Nanoparticulate preparations of poly (lactic-co-glycolic acid) (PLGA) are widely used due to their excellent drug loading capacity and are Food and Drug Administrationapproved materials. PLGA NPs are efficient carriers of drugs and biomolecules for the treatment of various ailments due to their biocompatibility and biodegradability. ${ }^{3}$ PLGA releases lactate on degradation, which promotes angiogenesis, stimulates collagen deposition, and promotes closure of wounds. ${ }^{4}$ Application of lactate to wounds has been reported to accelerate angiogenesis and the healing process. It can stimulate proliferation, migration, and capillary formation by endothelial cells. ${ }^{5-7}$ PLGA NP loaded with various factors like LL37 peptide, vascular endothelial growth factor (VEGF), and curcumin have been used to study the process of wound healing. ${ }^{8-10}$ To enable the simultaneous delivery of platelet-derived growth factor-BB and chlorhexidine, PLGA microspheres were developed. This, in turn, enabled the delivery of multiple cargoes. ${ }^{11}$

Chronic wounds have impaired wound healing and require prolonged healing time compared to acute wounds. ${ }^{12}$ The major causes of impairment in the healing of such wounds are ischemia and infection. Ischemia occurs due to the reduced levels of growth factors in the wound environment. ${ }^{13}$ VEGF and basic fibroblast growth factor (bFGF) promote wound healing by inducing angiogenesis and inhibiting prolonged inflammation. ${ }^{14}$ VEGF is upregulated in the early days of healing, leading to maximum capillary growth as VEGF enhances angiogenesis and mobilizes endothelial progenitors into the circulation. ${ }^{15,16} \mathrm{bFGF}$ also induces the proliferation of various cell types associated with the healing process, including fibroblasts and keratinocytes, which otherwise deteriorate in the wound location. ${ }^{17-19}$ The encapsulation of growth factors into NPs for delivery of drugs for healing helps to overcome problems of limited biodistribution, quick degradation, and clearance at the wound site. The small size and high surface-to-volume ratio of NPs enable to ease intracellular access and open up passages through the skin barrier. This is ideal for topical drug delivery.

Another factor influencing the process of wound healing is infection by microorganisms, prominently by bacteria that form biofilms in chronic wounds resulting in chronic inflammation and delay the healing process. ${ }^{20,21}$ Growing antimicrobial resistance is an important concern while treating bacterial infections. In the past few years, the focus of treatment has changed from antibiotic compounds to antimicrobial peptides (AMPs). AMPs are favored as they offer several advantages such as freedom of design and ease of synthesis due to solid phase peptide synthesis methods, and tools to predict the structure of the peptide and in turn assess the function of the designed peptide..$^{22-24}$

The main principle on which peptides have gained importance as antimicrobial agents is their capacity to penetrate cell membranes by forming $\alpha$ helical structure on account of amino acid arrangements. Based on the sequence and structure AMPs can be modulated to have lytic specificity toward prokaryotic cell membranes, thus sparing side effects on eukaryotic-mammalian cells. ${ }^{25}$ So we have preferred the use of AMP for addressing the infection aspect in the wound healing process. For the same, K4 peptide comprising 14 amino acids KKKKPLFGLFFGLF was selected from the AMP database whose broad-spectrum antimicrobial efficacy was validated by Duval et al. This peptide was selected as it is short and has the advantage of having a cationic end (ease of penetration in membrane based on positive charge), which adds to the $\alpha$ helical structure-based membrane penetration potential, as discussed earlier, gained on account of amphipathic helix due to the presence of hydrophobic amino acid residues such as leucine and phenylalanine. ${ }^{26}$

Both growth factors and peptide get degraded at a very high rate on topical application. Incorporation of these proteins into PLGA NP protects against enzymatic degradation, thus maintaining their integrity and activity as well as improving their bioavailability. ${ }^{27}$ In this study, we wanted to synthesize a system for codelivery of growth factors and AMP to address the major issues related to wound healing in chronic wounds. Hence, we have synthesized a dual growth factor delivery system by entrapping VEGF and bFGF in acid-terminated PLGA NPs by solvent diffusion method and conjugated an AMP (K4) to the PLGA NP by carbodiimide chemistry. This codelivery system was evaluated in vitro for its potential wound healing activity.

\section{Materials and methods \\ Materials}

PLGA(50:50, molecularweight 7,000-17,000 acid terminated), bFGF, VEGF, BSA, 1-ethyl-3-(3-dimethylaminopropyl) carbodiimide (EDC), N-hydroxysuccinimide (NHS), MTT, and lysozyme were purchased from Sigma, heparin and DCM were from Merk, and VEGF and bFGF ELISA kits were procured from $\mathrm{R}$ and D Biosystems. FBS was purchased from Pan America, and DMEM and live/dead assay kit were from Invitrogen.

\section{Peptide synthesis and purification}

The NH2-KKKKPLFGLFFGLF-COOH (K) peptide was synthesized by solid phase peptide synthesis using the 
standard Fmoc-Strategy. ${ }^{28,29}$ TentaGel ${ }^{\mathrm{TM}}-\mathrm{S}-\mathrm{NH} 2$ resin used was linked to HMPB linker by HBTU/HOBT activation, and peptide synthesis was carried out using the standard Fmoc methods. After the synthesis was completed, the peptide was manually cleaved using trifluoroacetic acid (TFA), triisopropylsilane, and water $(95: 2.5: 2.5)$. The peptide was then precipitated with diethyl ether, followed by 10-15 washes with diethyl ether. The peptide was dissolved in a minimum amount of water, lyophilized and stored at $-20^{\circ} \mathrm{C}$ after purification. The cleaved peptides were purified by high-performance liquid chromatography (HPLC) using a Shimadzu LC-AD HPLC system, equipped with a variablewavelength absorbance detector and a reverse phase $\mathrm{C} 18$ column. Buffer A containing water with $0.1 \%$ TFA and buffer B containing acetonitrile and water (80:20) containing $0.01 \%$ TFA at $1 \mathrm{~mL} / \mathrm{min}$ was used. The column eluents were monitored by ultraviolet absorbance at 214 and $254 \mathrm{~nm}$. Fractions were collected and lyophilized. The identity of the peptide was verified by matrix-assisted laser desorption ionization time-of-flight mass spectroscopy (MALDI-TOF MS).

\section{Preparation of growth factors-entrapped PLGA NPs}

VEGF- and bFGF-embedded PLGA NPs were synthesized using a modified version of solvent diffusion technique. ${ }^{30}$ Briefly, $100 \mathrm{mg}$ PLGA and $25 \mathrm{mg}$ pluronic F127 were dissolved in $3 \mathrm{~mL}$ of dichloromethane. To this, an aqueous solution containing $500 \mathrm{ng}$ VEGF, $500 \mathrm{ng}$ bFGF, $4 \mu \mathrm{g}$ heparin, and $200 \mu \mathrm{g}$ BSA was added. Heparin and BSA act as stabilizing agents. The solution was vortexed and poured into $25 \mathrm{~mL}$ of ethanol drop by drop under constant stirring. The solution was then diluted with $25 \mathrm{~mL}$ of water. The solvents were evaporated in vacuum. The NPs in aqueous medium were collected by centrifugation and lyophilized. All the above steps were similar for the synthesis of free NP except for the addition of growth factors.

\section{Conjugation of the K4 peptide to growth factor-entrapped PLGA NPs}

The purified K4 peptide was conjugated to growth factorentrapped PLGA NPs by EDC/NHS coupling. ${ }^{31}$ Ten milligrams of growth factor-entrapped PLGA NPs was dispersed in $1 \mathrm{~mL}$ of water containing $200 \mu \mathrm{L}$ of $40 \mathrm{mM}$ EDC and $200 \mu \mathrm{L}$ of $100 \mathrm{mM}$ NHS and incubated at room temperature for 1 hour with gentle shaking to activate the carboxyl group of PLGA NP. Twenty microliters of the peptide $(1 \mathrm{mg} / 1 \mathrm{~mL})$ was added to this solution and gently shaken for 2 hours at room temperature and then incubated at $4^{\circ} \mathrm{C}$ overnight.
The solution was then centrifuged for 15 minutes at 9,000 rpm and washed twice with distilled water. The pellet was lyophilized and stored at $-20^{\circ} \mathrm{C}$.

\section{Characterization TEM analysis}

Morphologic studies of the NPs were done by transmission electron microscopy (TEM, JEOL 1011, and Japan). The NPs were dissolved in Milli-Q water and dropped on copper-coated formvar microscopy grid. The sample was allowed to dry in a desiccator containing calcium chloride at room temperature. TEM pictures were taken using Digital Micrograph and Soft Imaging Viewer software.

\section{Particle size analysis}

The hydrodynamic diameter of PLGA NPs was measured by dynamic light scattering (DLS) method using Delsa ${ }^{\mathrm{TM}}$ Nanoparticle Size Analyzer (Beckman Coulter, Inc.) instrument.

\section{Zeta potential measurements}

The surface charge of the unconjugated and K4 conjugated NPs was analyzed by zeta potential measurements using Zetasizer Nano ZS (Malvern Instruments, Malvern, UK) instrument at ambient temperature.

\section{FT-IR spectroscopy}

The conjugation of the peptide to the PLGA NP was characterized by Fourier-transform infrared (FT-IR) spectroscopy (Perkin-Elmer, Lambda 25) fitted with an attenuated total reflectance mode cell. For each sample, eight scans were collected and averaged to reduce the signal-to-noise ratio. The spectral range was $4,000-600 \mathrm{~cm}^{-1}$.

\section{Entrapment efficiency of growth factors in PLGA NP}

The entrapment efficiency of the VEGF- and bFGF-loaded NPs was also evaluated by ELISA. The supernatants of the loaded NPs were collected and used to evaluate the amounts of VEGF and bFGF that were not encapsulated in NPs. The difference between the amount of VEGF and bFGF employed for the NP preparation, and the amount of VEGF and bFGF measured in the NP supernatant represented the amount of VEGF and bFGF loaded into the NPs. The encapsulation efficiency was calculated as a percentage of VEGF and bFGF loaded into the NPs compared to the total amount of VEGF, and bFGF added during the formulation of the NPs. 


\section{In vitro release study}

In vitro release of growth factors from dual growth factorloaded PLGA-K4 NPs was studied. Ten milligrams of growth factor-loaded PLGA NPs was suspended in $1 \mathrm{~mL}$ of PBS in a pretreated dialysis membrane of pore size $3,500 \mathrm{Da}$, which was immersed in $50 \mathrm{~mL}$ of PBS containing $1 \%$ BSA at $37^{\circ} \mathrm{C}$. One hundred microliters of samples was withdrawn at predetermined intervals and stored at $-20^{\circ} \mathrm{C}$. The amount of bFGF and VEGF released was determined by the Quantikine Immunoassay kits (ELISA) as per the manufacturer's instructions.

\section{Quantification of peptides conjugated to PLGA NPs}

The amount of peptide loaded onto the surface of PLGA NP was quantified by bicinchoninic acid (BCA) method. PLGA NPs suspended in water were taken as a blank (negative control), different concentrations of $\mathrm{K} 4$ peptide were taken, and BCA assay was performed to plot a standard curve. The amount of peptide conjugated was quantified by taking OD at $570 \mathrm{~nm}$ and was calculated by using the formulae Efficiency of conjugation $=($ Amount of conjugated peptide $/$ Total amount of peptide used for conjugation) $\times 100$.

\section{Cellular assays}

$\mathrm{HaCaT}$ cells were purchased from National Centre for Cell Science, Pune. The HaCaT cells were cultured in DMEM supplemented with $10 \%$ FBS and $1 \%$ antibiotics at $37^{\circ} \mathrm{C}$ under a humidified atmosphere containing $5 \%$ carbon dioxide. Until reaching $70 \%$ confluence in tissue culture flasks, the cells were trypsinized with PBS solution containing $0.25 \%$ trypsin and $0.03 \%$ EDTA.

\section{Cell viability assay}

Cell viability assay was performed by MTT reduction assay. $\mathrm{HaCaT}$ cells were seeded into 96 -well plate $\left(2 \times 10^{3}\right.$ cells/well $)$ and incubated under standard growth conditions for 24 hours. Cells were then treated with blank PLGA NPs $(1 \mathrm{mg} / \mathrm{mL})$, growth factors (40 $\mathrm{ng} / \mathrm{mL}$ each of VEGF and bFGF), K4 peptide $(89 \mu \mathrm{g} / \mathrm{mL})$, growth factor-entrapped PLGA NPs ( $1 \mathrm{mg} / \mathrm{mL}$ ), and peptide-conjugated PLGA NPs embedded with growth factors $(1 \mathrm{mg} / \mathrm{mL})$. After 24, 48, and 72 hours the cells were incubated for 4 hours with $10 \% \mathrm{v} / \mathrm{v}$ MTT ( $5 \mathrm{mg} / \mathrm{mL}$ dissolved in PBS $\mathrm{pH}$ 7.4). The medium was then removed and the formazan crystals thus formed were dissolved in isopropyl alcohol. Absorbance was measured at 570 nm using BioradiMark ${ }^{\mathrm{TM}}$ Microplate Absorbance Reader.
Percentage viability was calculated using the formula [(Avg. OD of test/Avg. OD of control) $\times 100]$.

\section{Live/dead assay}

Live/dead assay using calcein AM and ethidium homodimer fluorescent staining was performed to evaluate cell viability and proliferation of cells when in contact with the NP. HaCaT cells were seeded on a cover slip placed in a 12-well plate and medium containing the samples was added to it and incubated for 3 days. The concentration of the samples used was same as cell viability assay. The cells were then labeled with calcein AM and ethidium bromide homodimer-1 to distinguish the population of live cells from the dead cells. Images were acquired using a laser confocal microscope (TCS SP2; Leica Microsystems, Wetzlar, Germany).

\section{Scratch wound assay}

$\mathrm{HaCaT}$ cells were grown to form a confluent monolayer, which was then scratched with the $200 \mu \mathrm{L}$ pipette tip to create a uniform cell-free zone in each well. The wells were immediately washed with PBS to remove any cellular debris. The samples with same concentration as cell viability assay were added to the plate and incubated for 24 hours. The scratch wounds were observed at different time points after injury and pictures were taken, and the gap area between wound edges was measured at different time points with a computer-assisted analysis system (Olympus cellsens).

\section{Antimicrobial assay}

The antimicrobial activity of the peptide-conjugated NPs was calculated by the broth dilution method. Bacterial strains used for in vitro antibacterial assay were Staphylococcus aureus (Microbial Type Culture Collection and Gene Bank [MTCC] 9542) and Pseudomonas aeruginosa (MTCC 8076). They were purchased from MTCC, Chandigarh. Bacterial cultures were grown in Mueller-Hinton broth by overnight incubation at $37^{\circ} \mathrm{C}$ with constant shaking. The bacterial suspension was adjusted to the desired concentration by measuring the OD at $600 \mathrm{~nm}$ using a microplate reader. Then, $100 \mu \mathrm{L}$ of bacterial suspension $\left(1 \times 10^{6}\right.$ colony-forming units $\left./ \mathrm{mL}\right)$ and $100 \mu \mathrm{L}$ of sample solutions were added to a 96-well polypropylene plate and incubated at $37^{\circ} \mathrm{C}$ for 24 hours with shaking. OD at $600 \mathrm{~nm}$ was measured at 24 hours using a microplate reader to monitor bacterial growth.

\section{Statistical analysis}

All the measurements were performed in triplicate, and the results were expressed as an arithmetic mean \pm standard error 
of the mean. Statistical analysis of the data was assessed by Student's $t$-test. A value of significance $P<0.05$ was considered to be significant $(n=3)$.

\section{Results}

\section{Peptide synthesis and characterization}

The peptide K4 was manually synthesized with high yield and purity by solid phase peptide synthesis technique. The purity of the peptide is demonstrated in the HPLC profile by a single peak, with a retention time of 12.9 minutes (Figure 1B). The molecular weight of K4 peptide was determined by MALDI-TOF MS and gives a value of 1,670.831, which agrees with the calculated value of 1,670.1 (Figure 1A).

\section{NP synthesis and characterization}

Unloaded plain NPs and VEGF- and bFGF-entrapped biodegradable PLGA NPs were synthesized by a modified solvent diffusion technique as previously reported using Pluronic F127 as a stabilizer. ${ }^{30}$ The mean particle size of the NPs was determined by dynamic light scattering (DLS) and the size of growth factor-entrapped NP was found to be $416 \pm 6.9 \mathrm{~nm}$, which was slightly larger than the size of plain PLGA NP, which was $180 \pm 2.7 \mathrm{~nm}$, whereas TEM analysis showed that the size of the NPs was slightly smaller than the result obtained by DLS (Figure 2A-F). The differences observed in the two evaluations were essential because DLS measures the hydrodynamic radius of the particle due to the presence of NPs in a solvent whereas TEM was performed on dried NPs that do not have the presence of surface ions, solvent, and non-solvent molecules. ${ }^{32}$ The encapsulation efficiency of VEGF and bFGF in PLGA NP determined to be $75.98 \%$ and $83.65 \%$, respectively, was comparatively higher, which in turn minimizes the cost of production. The release kinetics of both VEGF and bFGF is affected by the diffusion of growth factors present on the surface of the NP, which showed an initial burst release followed by a gradual, sustained release, which corresponds to the release due to the degradation of PLGA NP (Figure 3).

\section{Peptide conjugation to NPs}

The amino group of the K4 peptide was successfully conjugated to the free carboxylic group of PLGA NP by EDC/ NHS coupling by the formation of an amide bond between PLGA NP and K4 peptide. The K4 peptide conjugated to the surface of PLGA NP was quantified by BCA method and was calculated to be $89 \%$. The interaction between the

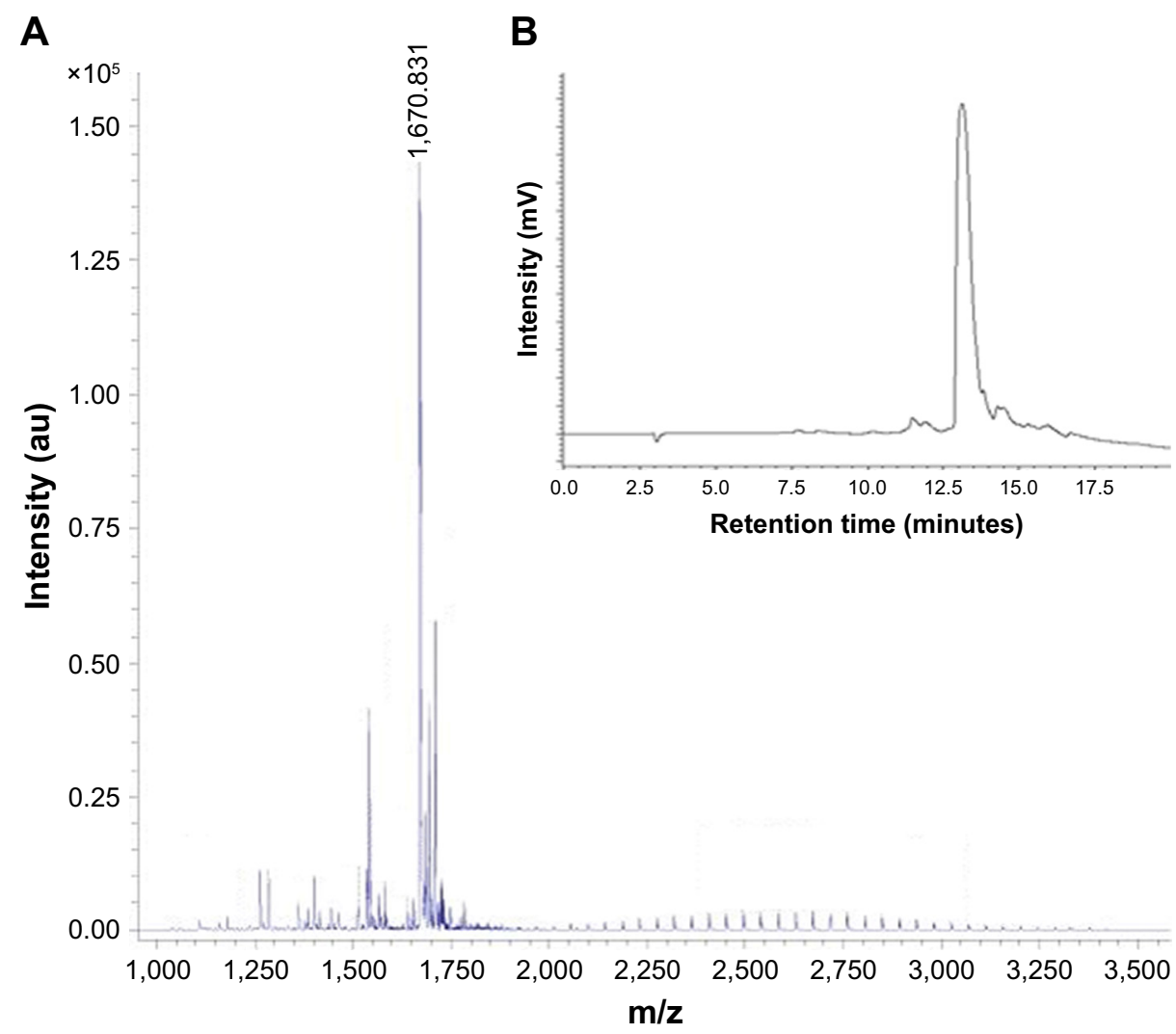

Figure I (A) Matrix-assisted laser desorption ionization time-of-flight mass spectroscopy showing mass of K4 as I,669.483. (B) High-performance liquid chromatography profile of K4. 
A
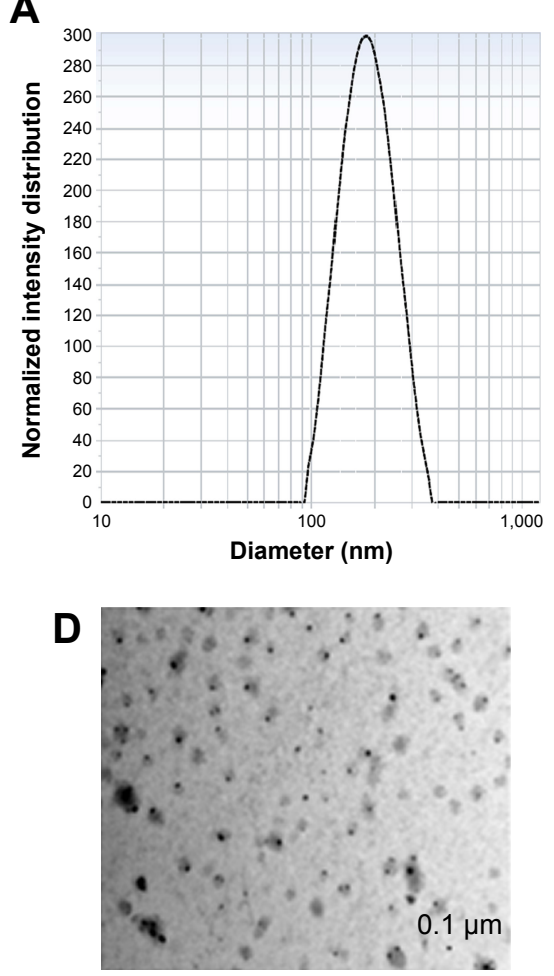

B
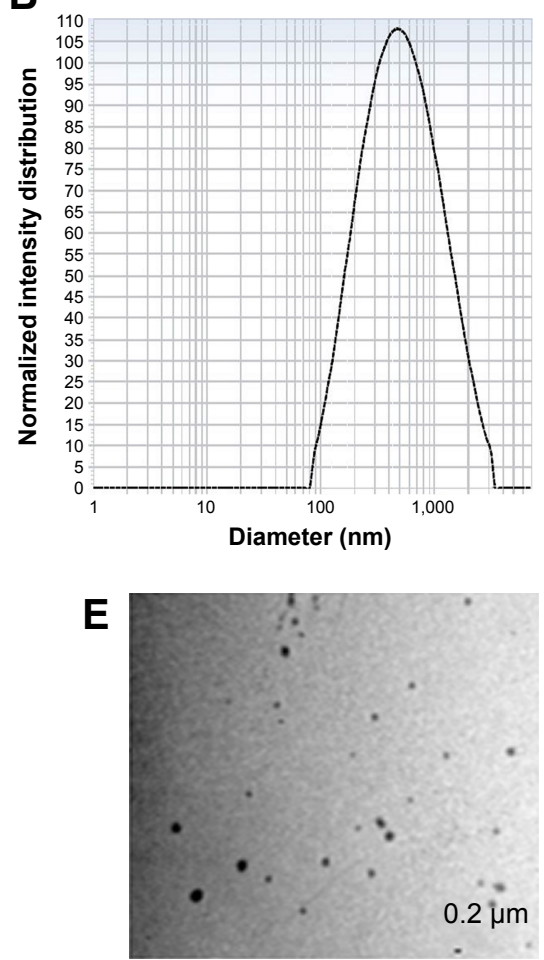

C

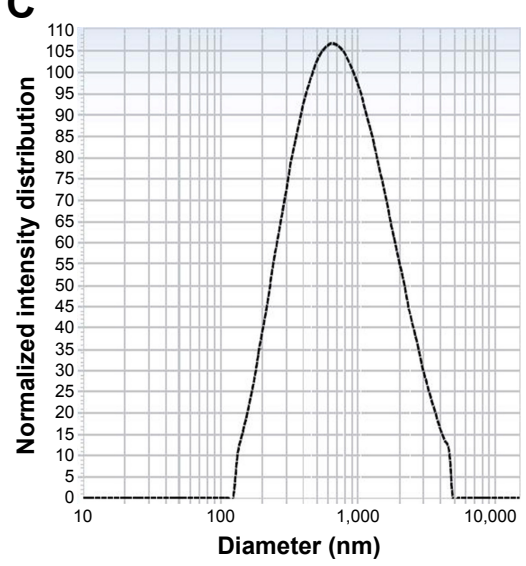

$\mathbf{F}$

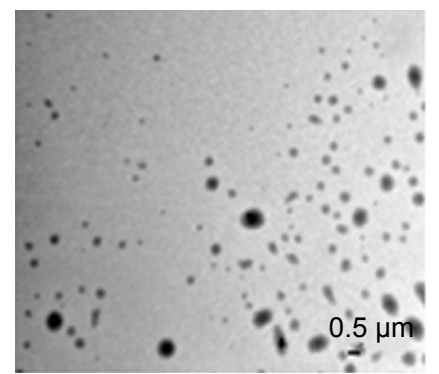

Figure 2 DLS measurement, nanoparticle size measurement of (A) PLGANP, (B) PLGA -GF NP, and (C) PLGA -GF -K4 NP and TEM images of (D) PLGANP, (E) PLGA -GF NP, and (F) PLGA -GF -K4 NP.

Abbreviations: DLS, dynamic light scattering; GF, growth factor; NP, nanoparticle; PLGA, poly (lactic-co-glycolic acid).

$\mathrm{NH} 2$ group of $\mathrm{K} 4$ peptide and $\mathrm{COOH}$ group of PLGA NP was confirmed by FT-IR analysis. Characteristic carbonyl peak of the carboxylic group at $1,754 \mathrm{~cm}^{-1}$ was present in the FT-IR spectra of unconjugated PLGA NP. Amide peaks at 1,630 and $1,561 \mathrm{~cm}^{-1}$ present in the K4-conjugated PLGA

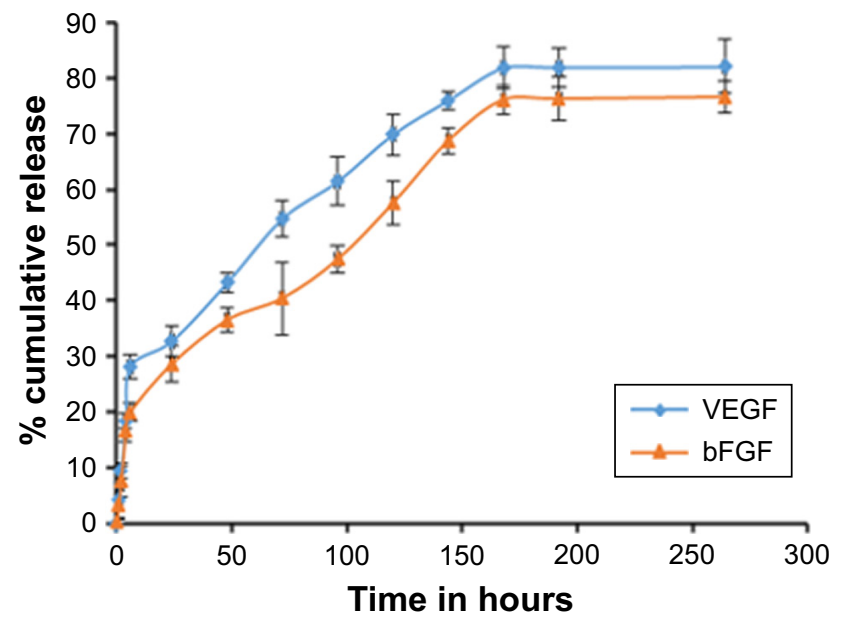

Figure 3 Release kinetics of bFGF and VEGF from poly (lactic-co-glycolic acid) nanoparticle (mean $\pm S D, n=3$ ).

Abbreviations: bFGF, basic fibroblast growth factor; VEGF, vascular endothelial growth factor.
NPs confirm the successful conjugation of K4 peptide to PLGA NPs (Figure 4).

The binding of the peptide was further characterized by the increase in the surface charge due to the presence of positively charged amino acids. There was a shift in zeta potential from -11 to 1 due to the conjugation of the K4 peptide. Due to the presence of lysine groups, the

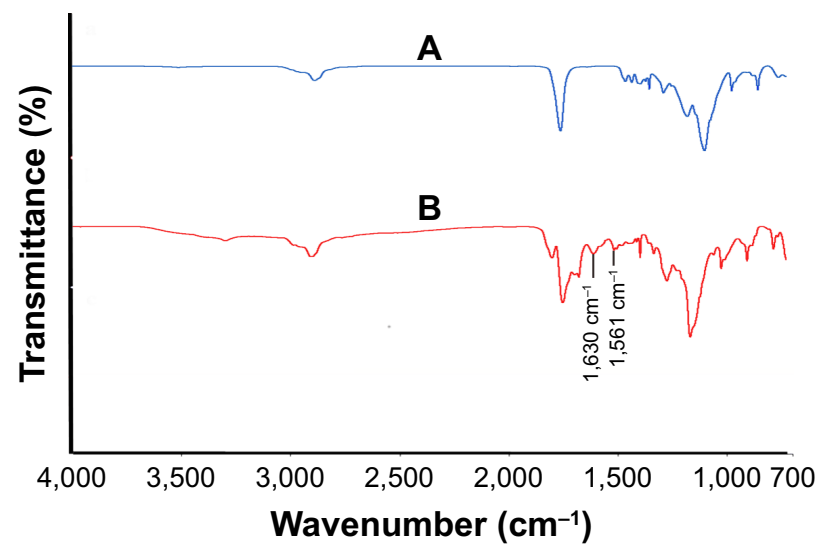

Figure 4 Fourier-transform infrared spectra of (A) unconjugated PLGA NP and (B) K4 peptide-conjugated PLGA NP.

Abbreviation: PLGA NP, poly (lactic-co-glycolic acid) nanoparticle. 
positive charge of the peptide decreases the zeta potential of the acid-terminated PLGA NPs upon conjugation to the peptide.

\section{Cytotoxicity assay}

MTT assay and live/dead assay were performed to investigate whether growth factor-entrapped PLGA NPs conjugated with $\mathrm{K} 4$ peptide were able to support $\mathrm{HaCaT}$ proliferation and survival. In MTT assay, cells were studied at 24,48 , and 72 hours intervals post sample administration, and it was found that on all the days studied, the growth factor-bound PLGA NPs exhibited high cell proliferation, whereas the growth factor-entrapped PLGA NPs conjugated with $\mathrm{K} 4$ peptide showed a small decrease in cell proliferation, which may be due to the presence of $\mathrm{K} 4$, which is a cationic AMP. Cell proliferation was significantly increased in all growth factor-entrapped NP-treated samples at 72 hours compared to the control, suggesting that the entrapment of growth factor preserved its bioactivity (Figure 5). The presence of heparin, which is used in the NP for growth factor stability, may also be responsible for its enhanced bioactivity. ${ }^{33,34}$ Relatively longer half-life of heparin compared to that of growth factors and its role in preserving the bioactivity of bound proteins against thermal and enzymatic degradation may be responsible for the above results. ${ }^{35} \mathrm{In}$ live/dead assay Calcein AM is retained within live cells, and red fluorescent dye EthD-1 enters cells through compromised cell membranes and binds to nucleic acids. The results were similar to that of MTT assay, showing that the PLGA-GF-K4 NP supported the proliferation and survival of $\mathrm{HaCaT}$ cells (Figure 6).

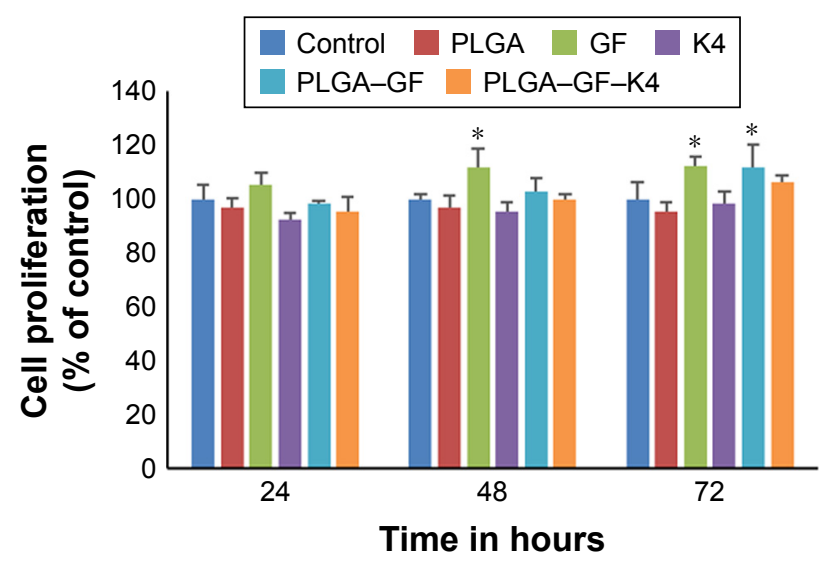

Figure 5 MTT assay of $\mathrm{HaCaT}$ cell proliferation treated with (1) control, (2) GF, (3) K4 peptide, (4) PLGA NP, (5) PLGA-GF NP, (6) PLGA-GF-K4 NP. Note: Values are represented as mean $\pm S D,(n=3, * P<0.05)$. Abbreviations: NP, nanoparticle; PLGA, poly (lactic-co-glycolic acid).

\section{Wound healing assay}

The effects of growth factors, K4 peptide, PLGA NP, PLGAGF NP, and PLGA-GF-K4 NP on the migratory capacity of $\mathrm{HaCaT}$ cells were determined by their ability to induce in vitro wound closure. The closure of wounds made in the confluent cell monolayers was tracked over 24 hours. K4 peptide and PLGA NP treatment did not seem to affect the migration rate of $\mathrm{HaCaT}$ cells, whereas cells treated with PLGA-GF NP showed a similar migration rate as those treated with free growth factor but faster than those treated with PLGA-GF-K4 NP (Figure 7).

\section{Antimicrobial assay}

The antimicrobial activity of the K4 peptide, PLGA, and PLGA-K4 was tested against gram-positive $S$. aureus and gram-negative $P$. aeruginosa. K4 peptide and PLGA-K4 NPs killed $\sim 75 \%$ and $\sim 40 \%$ of $S$. aureus (Figure $8 \mathrm{~A}$ ) and $\sim 50 \%$ and $\sim 30 \%$ of $P$. aeruginosa (Figure $8 \mathrm{~B}$ ), respectively, indicating that our system has a broad spectrum of antibacterial activity. PLGA NP did not show any significant antimicrobial activity (Figure 8).

\section{Discussion}

Chronic wounds remain in a pathologic inflammatory state, which provides a proteolytic microenvironment at the wound site leading to the degradation of growth factors, which delays or impairs the normal wound healing process. These types of wounds have also exhibited high bacterial load, which can further delay the healing process. ${ }^{36}$ Clinical trials of wound healing through topical application of growth factors were largely unsuccessful due to their rapid degradation and extremely short half-life. ${ }^{37}$ PLGA NP has been used successfully as a growth factor delivery system due to its biocompatibility and degradability. ${ }^{38,39}$ In the present paper, a drug delivery system using PLGA NP for the codelivery of growth factors and the AMP was developed. Dual growth factor-encapsulated NPs were prepared by modified solvent diffusion technique using poloxamer F127 as a stabilizer, which gave rise to NPs with a size of $416 \mathrm{~nm}$. Presence of pluronic in the PLGA nanomatrix has been reported to avoid the burst release of protein from PLGA micro- and nanospheres. ${ }^{37}$ Growth factor degradation and inactivation are caused by elevated levels of matrix metalloproteinase and other factors at the site of the wound. ${ }^{40}$ Addition of heparin and BSA during the synthesis of the NP helps in stabilizing the growth factors against enzymatic degradation and thermal instability. ${ }^{41,42}$ The growth factors VEGF and bFGF were entrapped in the PLGA NP with high entrapment efficiency and a sustained release over a prolonged period after 

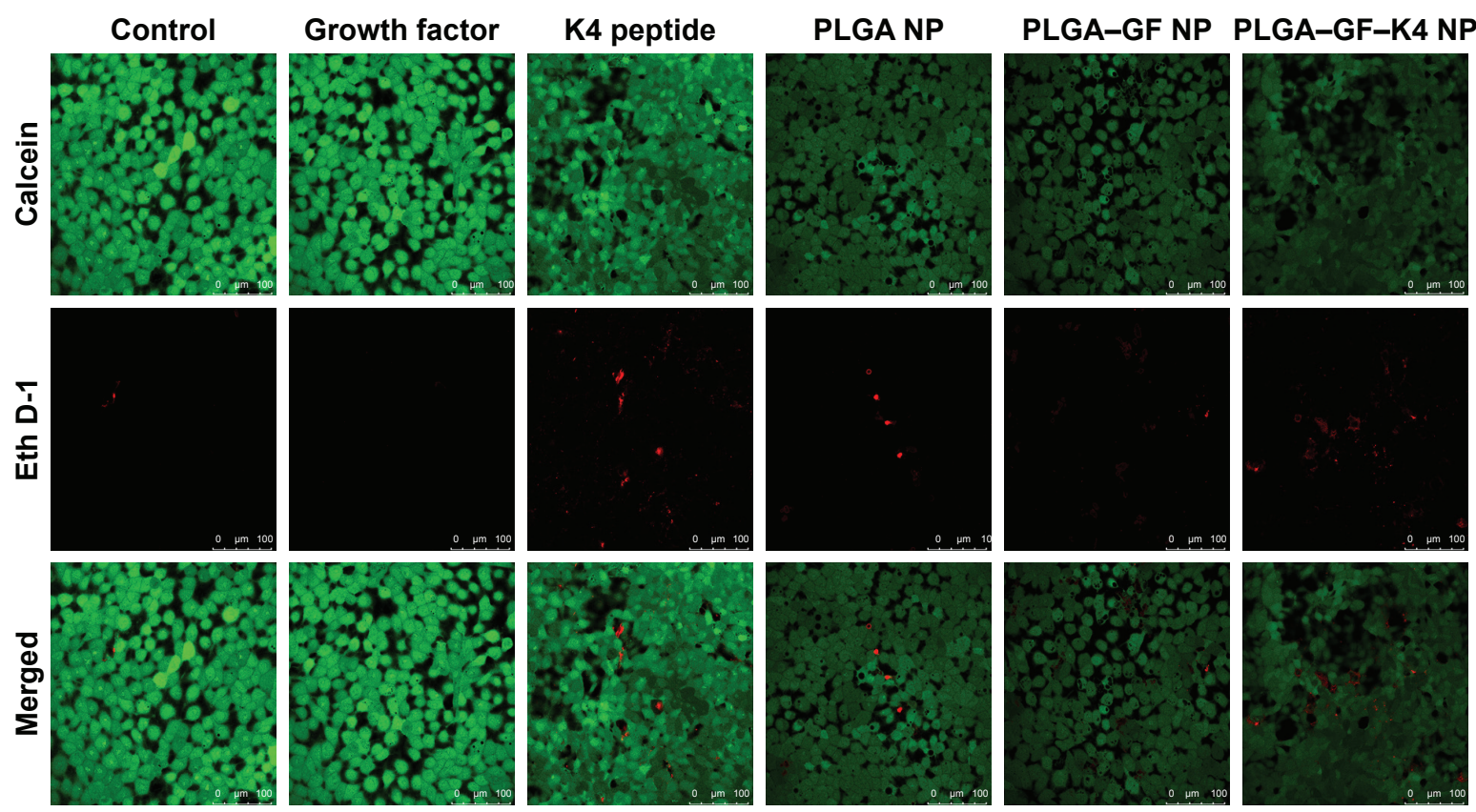

Figure 6 Live/dead cell assay of HaCaT cells treated with (I) control, (2) GF, (3) K4 peptide, (4) PLGA NP, (5) PLGA-GF NP, and (6) PLGA-GF-K4 NP. Note: Magnification: $20 \times$ and scale bar is $100 \mu \mathrm{m}$.

Abbreviations: NP, nanoparticle; PLGA, poly (lactic-co-glycolic acid).

an initial burst release was observed. For effective healing of complex wounds, gradual release of growth factors in a sustained manner has been reported to be effective, which was evaluated by Quantikine ELISA.

Skin wounds are colonized by opportunistic pathogens like $P$. aeruginosa and $S$. aureus forming biofilms. AMPs do not induce resistance in microbes compared to conventional antibiotics as they disintegrate the microbial cell membrane killing the bacteria by electrostatic interaction between positive amino acid residues of AMP and negative charges exposed on bacterial cell surfaces. ${ }^{43,44}$ We have synthesized a synthetic cationic AMP (K4) that was conjugated to the growth factor-entrapped PLGA NP. K4 was reported to have a broad spectrum of antibacterial activity and destabilizes the bacterial membranes by a detergent-like mechanism. ${ }^{45}$ The conjugation of peptide to the PLGA NP by carbodiimide chemistry was observed by FT-IR and also zeta potential measurement. The efficiency of peptide conjugation to the NP was quantified by BCA assay.

The growth factor released from the nanoparticulate system was tested for its bioactivity by cell viability assay and live/dead assay. The cells treated with the growth factor-entrapped PLGA NP showed increased proliferation compared to free growth factor-treated cells, which indicate
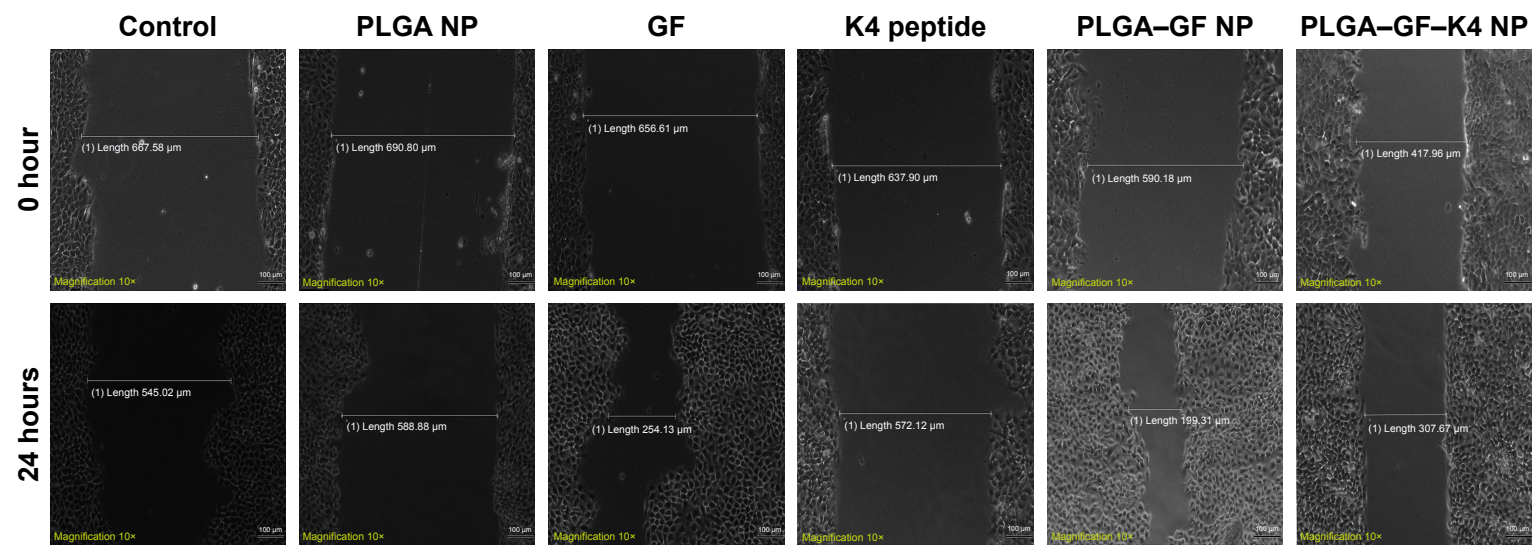

Figure 7 In vitro migration assay.

Note: Images were obtained at 0 and 24 hours after wound creation. Magnification: 10× and scale bar is $100 \mu \mathrm{m}$.

Abbreviations: NP, nanoparticle; PLGA, poly (lactic-co-glycolic acid). 


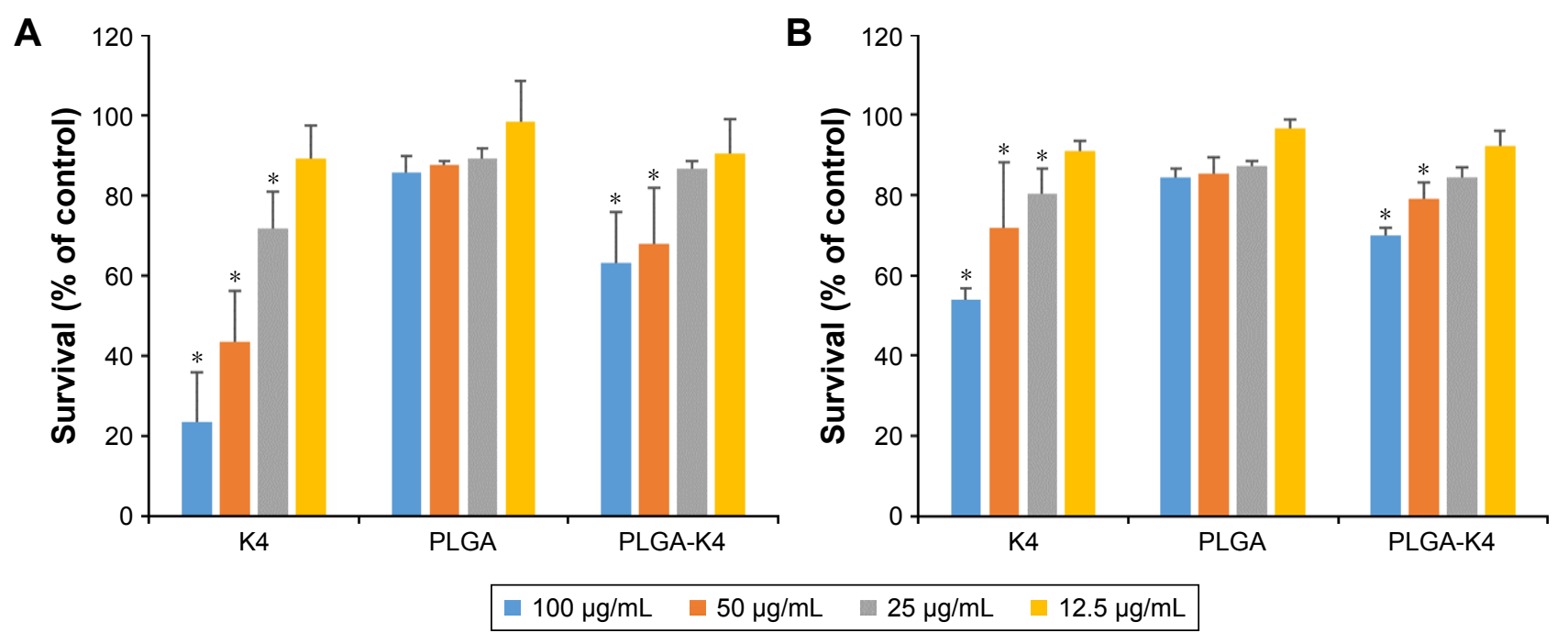

Figure 8 Antimicrobial activity of K4 peptide, PLGA NP, and PLGA-K4 NP against (A) Staphylococcus aureus and (B) Pseudomonas aeruginosa. Values are represented as mean $\pm S D(n=3, * P<0.05)$. Statistical significance compared with untreated group.

Abbreviations: NP, nanoparticle; PLGA, poly (lactic-co-glycolic acid).

the increased stability and bioactivity of growth factors encapsulated by PLGA NPs. The K4 peptide-conjugated growth factor-loaded NPs showed only a slight difference in activity compared to growth factor-loaded NPs, which established that $\mathrm{K} 4$ is not toxic to mammalian cells at lower concentrations. ${ }^{30}$ The PLGA-GF-K4 NP treatment also leads to increased migration of keratinocytes compared to control cells, indicating enhanced wound healing in vitro. Though the attachment of K4 to the PLGA NP affected the efficiency of its antibacterial activity, it still shows a significant reduction in the bacterial load of both $S$. aureus and $P$. aeruginosa at concentrations of 50 and $100 \mu \mathrm{g} / \mathrm{mL}$ when compared to the untreated group. This proves that the PLGA-GF-K4 NP has a broad range of antibacterial activity.

In chronic wounds, infection caused due to microbial factors seriously affects the healing process. ${ }^{20}$ Emerging treatments using AMPs show promising results in decreasing the microbial load and its immunomodulatory activities favoring healing. ${ }^{22}$ In our multiple cargo delivery system, $\mathrm{K} 4$ peptide acts as an antimicrobial agent to reduce wound infection and the growth factors used will further enhance the healing in chronic wounds. K4 peptide though reported to be noncytotoxic at low concentrations, can cause mammalian cell death as the concentration increases. We have tried to keep the concentration of $\mathrm{K} 4$ as low as possible without affecting its antibacterial activity. As seen from the MTT results PLGA-GF-K4 particle does not show significant toxicity compared to PLGA-GF NPs. During in vitro studies, even the dual delivery system as such may show very low toxicity. When viewing it in a chronic wound healing perspective, the significantly low toxicity of the system is bound to be negligible. This system can be used as a potential healing system for chronic wounds by addressing the dual problems of ischemia and infection.

\section{Conclusion}

The development of a system for the codelivery of growth factors (VEGF and bFGF) and an AMP (K4) was investigated for potential wound healing application. The entrapment of growth factors with very high efficiency is an advantage in this method along with its sustained release from the nanoparticulate system, which will enhance the angiogenesis. The AMP K4 was immobilized onto the surface of growth factor-entrapped PLGA NP to protect the wound against infection, thus addressing the major problems of chronic wounds. The system enhanced the proliferation of $\mathrm{HaCaT}$ cells and also increased its rate of migration. These results indicate the potential of K4-conjugated PLGA-GF NP system as a wound healing system.

\section{Acknowledgments}

The authors are grateful to Mr Anurup KG of the Rajiv Gandhi Centre for Biotechnology for confocal laser scanning microscopy analysis; the Department of Biotechnology, New Delhi, for providing the financial assistance for the research work; the University Grants Commission, New Delhi, for the Junior Research Fellowship to Amritha Vijayan. Rajiv Gandhi Centre for Biotechnology is affiliated to Kerala University for $\mathrm{PhD}$ registration.

\section{Disclosure}

The authors report no conflicts of interest in this work. 


\section{References}

1. Berthet M, Gauthier Y, Lacroix C, Verrier B, Monge C. Nanoparticlebased dressing: the future of wound treatment? Trends Biotechnol. 2017;35(8):770-784.

2. Pachuau L. Recent developments in novel drug delivery systems for wound healing. Expert Opin Drug Deliv. 2015;12(12):1895-1909.

3. Nair KL, Jagadeeshan S, Nair SA, Kumar GS. Biological evaluation of 5-fluorouracil nanoparticles for cancer chemotherapy and its dependence on the carrier, PLGA. Int J Nanomedicine. 2011;6:1685-1697.

4. Song R, Murphy M, Li C, Ting K, Soo C, Zheng Z. Current development of biodegradable polymeric materials for biomedical applications. Int J Nanomed. 2018;12:3117-3145.

5. Beckert S, Farrahi F, Aslam RS, et al. Lactate stimulates endothelial cell migration. Wound Repair Regen. 2006;14(3):321-324.

6. Milovanova TN, Bhopale VM, Sorokina EM, et al. Lactate stimulates vasculogenic stem cells via the thioredoxin system and engages an autocrine activation loop involving hypoxia-inducible factor $1 \mathrm{Mol}$ Cell Biol. 2008;28(20):6248-6261.

7. Jain RA. The manufacturing techniques of various drug loaded biodegradable poly(lactide-co-glycolide) (PLGA) devices. Biomaterials. 2000;21(23):2475-2490.

8. Chereddy KK, Her CH, Comune M, et al. PLGA nanoparticles loaded with host defense peptide LL37 promote wound healing. J Control Release. 2014;194:138-147.

9. Chereddy KK, Lopes A, Koussoroplis S, et al. Combined effects of PLGA and vascular endothelial growth factor promote the healing of nondiabetic and diabetic wounds. Nanomedicine. 2015;11(8):1975-1984.

10. Chereddy KK, Coco R, Memvanga PB, et al. Combined effect of PLGA and curcumin on wound healing activity. J Control Release. 2013; 171(2):208-215

11. Jiang B, Zhang G, Brey EM. Dual delivery of chlorhexidine and plateletderived growth factor-BB for enhanced wound healing and infection control. Acta Biomater. 2013;9(2):4976-4984.

12. Wysocki AB. Wound fluids and the pathogenesis of chronic wounds. J Wound Ostomy Continence Nurs. 1996;23(6):283-290.

13. Harding KG, Morris HL, Patel GK. Science, medicine and the future: healing chronic wounds. BMJ. 2002;324(7330):160-163.

14. Barrientos S, Stojadinovic O, Golinko MS, Brem H, Tomic-Canic M. Growth factors and cytokines in wound healing. Wound Repair Regen. 2008;16(5):585-601.

15. Galiano RD, Tepper OM, Pelo CR, et al. Topical vascular endothelial growth factor accelerates diabetic wound healing through increased angiogenesis and by mobilizing and recruiting bone marrow-derived cells. Am J Pathol. 2004;164(6):1935-1947.

16. Bao P, Kodra A, Tomic-Canic M, Golinko MS, Ehrlich HP, Brem H. The role of vascular endothelial growth factor in wound healing. J Surg Res. 2009;153(2):347-358.

17. Werner S, Grose R. Regulation of wound healing by growth factors and cytokines. Physiol Rev. 2003;83(3):835-870.

18. Mccarty SM, Percival SL. Proteases and delayed wound healing. Adv Wound Care. 2013;2(8):438-447.

19. Liu J, Zheng H, Dai X, Sun S, Machens HG, Schilling AF. Biomaterials for promoting wound healing in diabetes. J Tissue Sci Eng. 2017; 8(1):193-196.

20. Rhoads DD, Wolcott RD, Percival SL. Biofilms in wounds: management strategies. J Wound Care. 2008;17(11):502-508.

21. Lipsky BA, Hoey C. Topical antimicrobial therapy for treating chronic wounds. Clin Infect Dis. 2009;49(10):1541-1549.

22. Hancock RE, Sahl HG. Antimicrobial and host-defense peptides as new anti-infective therapeutic strategies. Nat Biotechnol. 2006; 24(12):1551-1557.

23. Marr AK, Gooderham WJ, Hancock RE. Antibacterial peptides for therapeutic use: obstacles and realistic outlook. Curr Opin Pharmacol. 2006;6(5):468-472.

24. Galdiero S, Falanga A, Berisio R, Grieco P, Morelli G, Galdiero M. Antimicrobial peptides as an opportunity against bacterial diseases. Curr Med Chem. 2015;22(14):1665-1677.
25. Blondelle SE, Lohner K. Combinatorial libraries: a tool to design antimicrobial and antifungal peptide analogues having lytic specificities for structure-activity relationship studies. Biopolymers. 2000;55(1):74-87.

26. Duval E, Zatylny C, Laurencin M, Baudy-Floc'h M, Henry J. KKKKPLFGLFFGLF: a cationic peptide designed to exert antibacterial activity. Peptides. 2009;30(9):1608-1612.

27. Danhier F, Ansorena E, Silva JM, Coco R, Le Breton A, Préat V. PLGA-based nanoparticles: an overview of biomedical applications. J Control Release. 2012;161(2):505-522.

28. Behrendt R, White P, Offer J. Advances in Fmoc solid-phase peptide synthesis. J Pept Sci. 2016;22(1):4-27.

29. Ashwanikumar N, Kumar NA, Nair SA, Kumar GSV. Phenylalaninecontaining self-assembling peptide nanofibrous hydrogel for the controlled release of 5-fluorouracil and leucovorin. RSC Advances. 2014; 4(55):29157-29164.

30. Csaba N, González L, Sánchez A, Alonso MJ. Design and characterisation of new nanoparticulate polymer blends for drug delivery. J Biomater Sci Polym Ed. 2004;15(9):1137-1151.

31. Mathew A, Fukuda T, Nagaoka Y, et al. Curcumin loaded-PLGA nanoparticles conjugated with Tet-1 peptide for potential use in Alzheimer's disease. PLoS One. 2012;7(3):e32616.

32. Zanetti-Ramos BG, Fritzen-Garcia MB, de Oliveira CS, et al. Dynamic light scattering and atomic force microscopy techniques for size determination of polyurethane nanoparticles. Mater Sci Eng C. 2009; 29(2):638-640.

33. Ashikari-Hada S, Habuchi H, Kariya Y, Kimata K. Heparin regulates vascular endothelial growth factor 165-dependent mitogenic activity, tube formation, and its receptor phosphorylation of human endothelial cells. Comparison of the effects of heparin and modified heparins. J Biol Chem. 2005;280(36):31508-31515.

34. Nugent MA, Iozzo RV. Fibroblast growth factor-2. Int J Biochem Cell Biol. 2000;32(2):115-120.

35. Brandner B, Kurkela R, Vihko P, Kungl AJ. Investigating the effect of VEGF glycosylation on glycosaminoglycan binding and protein unfolding. Biochem Biophys Res Commun. 2006;340(3):836-839.

36. Gainza G, Villullas S, Pedraz JL, Hernandez RM, Igartua M. Advances in drug delivery systems (DDSs) to release growth factors for wound healing and skin regeneration. Nanomed Nanotechnol. 2015;11(6): 1551-1573.

37. Chen RR, Mooney DJ. Polymeric growth factor delivery strategies for tissue engineering. Pharm Res. 2003;20(8):1103-1112.

38. Liu Y, Wu X, Mi Y, et al. PLGA nanoparticles for the oral delivery of nuciferine: preparation, physicochemical characterization and in vitro/ in vivo studies. Drug Deliv. 2017;24(1):443-451.

39. Ru G, Han L, Qing J, et al. Effects of borneol on the pharmacokinetics of 9-nitrocamptothecin encapsulated in PLGA nanoparticles with different size via oral administration. Drug Deliv. 2016;23(9):3417-3423.

40. Trengove NJ, Stacey MC, Macauley S, et al. Analysis of the acute and chronic wound environments: the role of proteases and their inhibitors. Wound Repair Regen. 1999;7(6):442-452.

41. King TW, Patrick CW. Development and in vitro characterization of vascular endothelial growth factor (VEGF)-loaded poly(DL-lacticco-glycolic acid)/poly(ethylene glycol) microspheres using a solid encapsulation/single emulsion/solvent extraction technique. J Biomed Mater Res. 2000;51(3):383-390.

42. Joung YK, Bae JW, Park KD. Controlled release of heparin-binding growth factors using heparin-containing particulate systems for tissue regeneration. Expert Opin Drug Deliv. 2008;5(11):1173-1184.

43. Mangoni ML, Mcdermott AM, Zasloff M. Antimicrobial peptides and wound healing: biological and therapeutic considerations. Exp Dermatol. 2016;25(3):167-173.

44. Guilhelmelli F, Vilela N, Albuquerque P, Derengowski LS, SilvaPereira I, Kyaw CM. Antibiotic development challenges: the various mechanisms of action of antimicrobial peptides and of bacterial resistance. Front Microbiol. 2013;4:353.

45. Legrand B, Laurencin M, Sarkis J, et al. Structure and mechanism of action of a de novo antimicrobial detergent-like peptide. Biochim Biophys Acta. 2011;1808(1):106-116. 
International Journal of Nanomedicine

Dovepress

\section{Publish your work in this journal}

The International Journal of Nanomedicine is an international, peerreviewed journal focusing on the application of nanotechnology in diagnostics, therapeutics, and drug delivery systems throughout the biomedical field. This journal is indexed on PubMed Central, MedLine, CAS, SciSearch $®$, Current Contents $\circledR /$ Clinical Medicine,
Journal Citation Reports/Science Edition, EMBase, Scopus and the Elsevier Bibliographic databases. The manuscript management system is completely online and includes a very quick and fair peer-review system, which is all easy to use. Visit http://www.dovepress.com/ testimonials.php to read real quotes from published authors.

Submit your manuscript here: http://www.dovepress.com/international-journal-of-nanomedicine-journal 